\title{
Patent ductus arteriosus in adults - indications and possibilities for closure
}

\author{
F. M. van de Sandt • S. M. Boekholdt • B. J. Bouma • \\ M. Groenink • A. P. C. M. Backx • D. R. Koolbergen • \\ R. J. de Winter $\cdot$ B. J. M. Mulder
}

Published online: 17 May 2011

(C) The Author(s) 2011. This article is published with open access at Springerlink.com

\begin{abstract}
Patent ductus arteriosus (PDA) is a rare diagnosis in adults, since symptoms and signs usually occur in infancy and most cases are treated shortly after diagnosis. We present two patients who were first diagnosed with PDA during adulthood. The first case represents a more severe form of PDA, where the need for closure of the PDA is obvious. In the second case the sequelae of the PDA are less clear. In both patients, closure of the PDA (surgically in one case, percutaneously in the other) was successful.
\end{abstract}

Keywords Patent ductus arteriosus - Open ductus Botalli . Congenital heart disease - Percutaneous closure .

Pulmonary hypertension

F. M. van de Sandt

Department of Cardiology, Hofpoort Hospital,

PO Box 8000, 3440 JD Woerden, the Netherlands

S. M. Boekholdt $\cdot$ B. J. Bouma $\cdot$ M. Groenink

A. P. C. M. Backx • R. J. de Winter · B. J. M. Mulder $(\bowtie)$

Department of Cardiology, Academic Medical Center,

PO Box 22660, 1100 DD Amsterdam, the Netherlands

e-mail: b.j.mulder@amc.uva.nl

A. P. C. M. Backx

Department of Pediatric Cardiology, Academic Medical Center,

PO Box 22660, 1100 DD Amsterdam, the Netherlands

D. R. Koolbergen

Department of Pediatric Cardiac Surgery,

Academic Medical Center,

PO Box 22660, 1100 DD Amsterdam, the Netherlands

D. R. Koolbergen

Department of Cardiothoracic Surgery,

Leiden University Medical Center,

PO Box 9600, 2300 RC Leiden, the Netherlands
Patent ductus arteriosus (PDA) represents 5 to $10 \%$ of all congenital heart defects [1]. It can be associated with other cardiovascular anomalies, most importantly coarctation of the aorta, ventricular septal defect and aortic or pulmonary stenosis. PDA is most often diagnosed and surgically or percutaneously corrected in infancy, so a primary diagnosis is rare in adulthood. Left untreated, PDA can lead to pulmonary hypertension, Eisenmenger syndrome, heart failure and endarteritis. We report two adult patients diagnosed with PDA.

\section{Case A}

A 20-year-old refugee from Liberia was referred to our hospital because of a loud murmur. He had been suffering from fatigue and mild exertional dyspnoea. In the past, he had been admitted to a hospital in his home country several times because of respiratory tract infections and heart problems'. He had a low body weight of $45 \mathrm{~kg}$ at a height of $1.63 \mathrm{~m}$. He was hypertensive with a $50 \mathrm{mmHg}$ difference in systolic blood pressure between his upper and lower extremities: 170 vs. $120 \mathrm{mmHg}$. Diastolic blood pressure was immeasurable. A loud continuous murmur was heard on the chest and back. Physical examination did not reveal additional abnormalities. The electrocardiogram showed sinus rhythm 84 beats/min with signs of left ventricular hypertrophy.

Echocardiography (Fig. 1) revealed a dilated, hypertrophied and hyperdynamic left ventricle due to a large PDA with a continuous left-to-right shunt. Furthermore, a stenosis of the proximal right pulmonary artery was present. Just distal to the PDA, narrowing of the aorta was visible, with an increased systolic flow velocity. The absence of a classic 'sawtooth' pattern in the continuous wave signal 


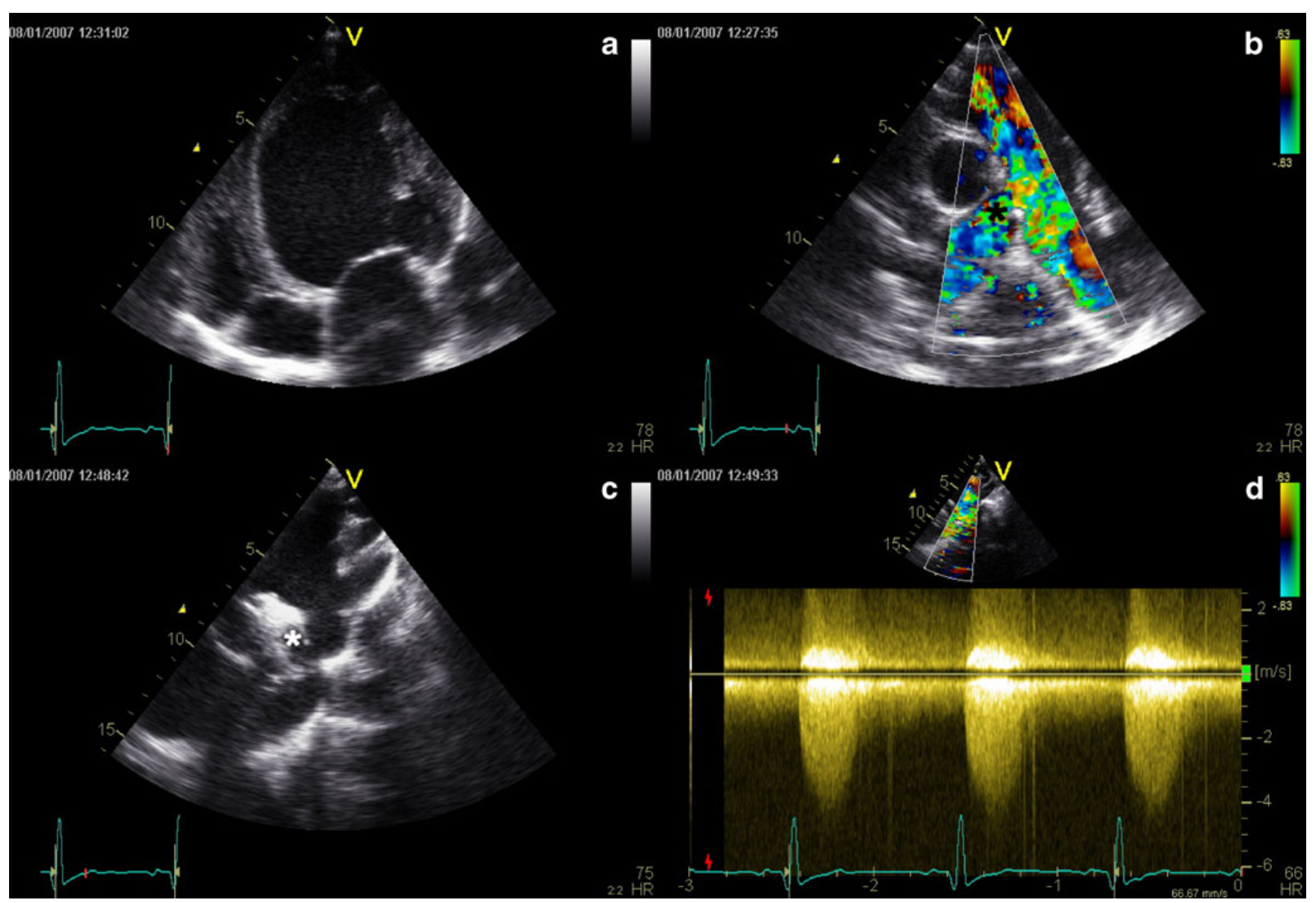

Fig. 1 Transthoracic echocardiography in patient A. a Apical fourchamber view showing dilated and hypertrophied left ventricle. b Parasternal view of the pulmonary trunk and arteries showing turbulent flow due to a PDA with left-to-right shunting; a stenosis of the proximal right pulmonary artery is also visible (asterisk). c
Suprasternal view showing PDA (asterisk) as well as coarctation of the aorta. d Continuous wave Doppler signal from the descending aorta, showing elevated systolic flow velocity of about $4 \mathrm{~m}$ per second. A typical 'sawtooth' pattern is lacking
Fig. 2 Preoperative and postoperative magnetic resonance angiography. a Preoperative magnetic resonance angiography (MRA) of the aorta (sagittal plane), showing large PDA (black arrow) and irregular aspect of the descending aorta with coarctation distal to the PDA (white arrow). b Repeat MRA of the aorta after surgical correction of the PDA, showing irregular aspect and (mild) residual stenosis/coarctation of the descending aorta

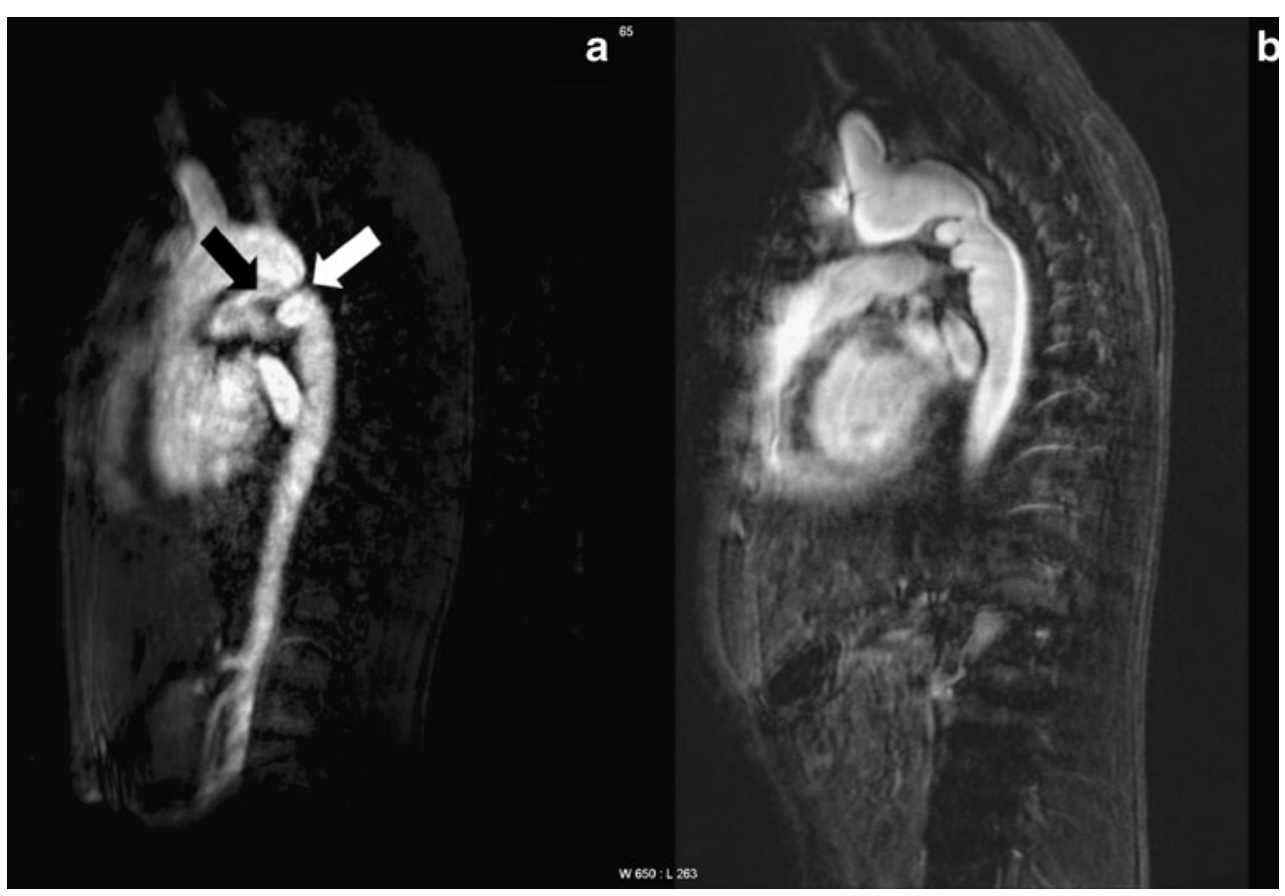


from the descending aorta as well as the presence of holodiastolic flow reversal in the abdominal aorta suggested non-severe coarctation. The cardiac valves showed no abnormalities except for a mild pulmonary regurgitation with an early-diastolic flow velocity of around $3 \mathrm{~m}$ per second, suggestive of elevated pulmonary artery pressure. Magnetic resonance imaging (MRI, Fig. 2a) confirmed these findings. The large PDA was visualised. The descending thoracic aortic wall had an irregular aspect and its narrowing was most severe just distal to the PDA. The left ventricular ejection fraction was calculated at $52 \%$. Right heart catheterisation was performed. Right ventricular and main pulmonary artery pressures were elevated (systolic right ventricular pressure $55 \mathrm{mmHg}$, main pulmonary artery pressure $48 / 31 \mathrm{mmHg}$ at a systemic blood pressure of $167 / 84 \mathrm{mmHg}$ ), but peripheral pulmonary artery pressures were normal. A peak-to-peak gradient of $50 \mathrm{mmHg}$ was measured in the descending aorta.

The patient was referred for surgery. Closure of the ductus arteriosus was performed and the pulmonary artery was reconstructed (Fig. 2b). The right pulmonary artery stenosis was treated with a pericardial patch. After ligation of the PDA, the pressure gradient in the descending aorta had decreased from 50 to $20 \mathrm{mmHg}$ and this (mild) residual coarctation was left untreated. Whether it will need (percutaneous) treatment in the future remains to be seen.

\section{Case B}

A 55-year-old male was referred to our hospital for treatment of PDA. He had previously been diagnosed with severe hypertension with a poor response to medical therapy. Approximately eight years prior to referral to our hospital, echocardiography had already revealed left ventricular hypertrophy. Moreover, it showed a PDA with a continuous left-to-right shunt. A murmur had never been heard. At that time there was no left ventricular dilatation and right ventricular systolic pressure was normal. An MRA did not reveal coarctation of the aorta or renal artery stenosis. The patient was kept under annual surveillance.

When he was referred to our outpatient clinic he did not have any cardiac complaints, although his exercise capacity had gradually deteriorated over the past year. In addition to the PDA, echocardiography now showed not only left, but also right ventricular hypertrophy and an estimated right ventricular systolic pressure of $57 \mathrm{mmHg}$. MRI visualised the flow through the PDA (Fig. 3). He was referred for treatment of his PDA on the assumption that it had contributed to the development of pulmonary hypertension. It must be noted that the PDA was silent and did not appear very large on echocardiographic and MRI. Moreover,

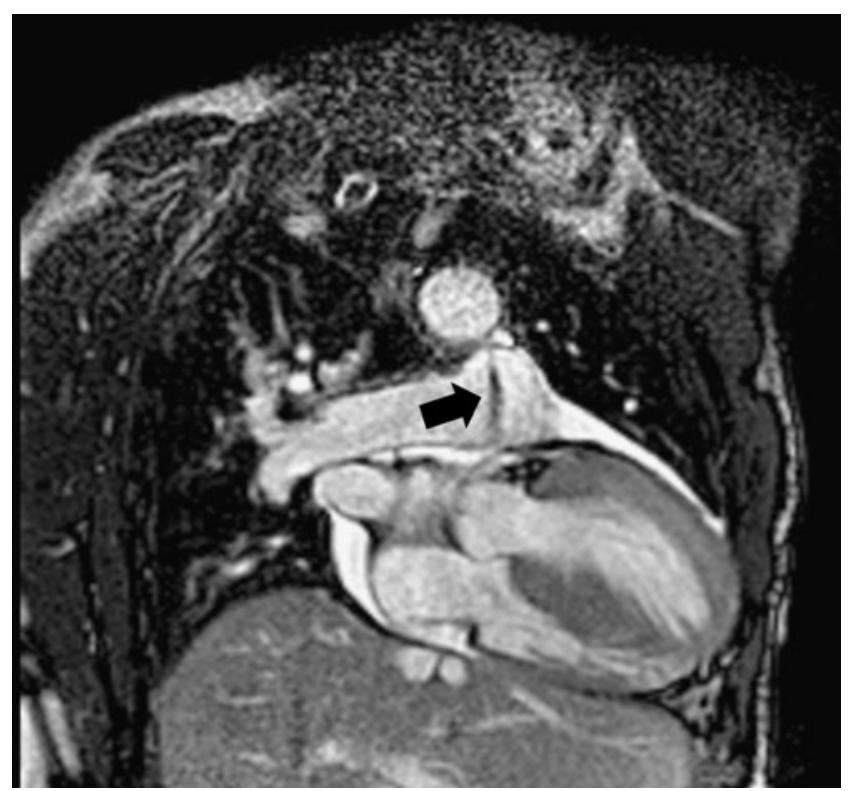

Fig. 3 Magnetic resonance imaging of PDA. MRI visualised the jet of the PDA flow entering the pulmonary bifurcation (black arrow)

diastolic dysfunction due to left ventricular hypertrophy may have played an important role in the development of pulmonary hypertension as well. However, a contribution of the PDA to the development of pulmonary hypertension could not be excluded and the patient was therefore accepted for percutaneous closure of the PDA. The procedure was successful. Angiography of the aortic arch and the occluded PDA with coil in situ can be seen in Fig. 4. In due time, echocardiography will be repeated to document whether his pulmonary artery pressure will decrease.

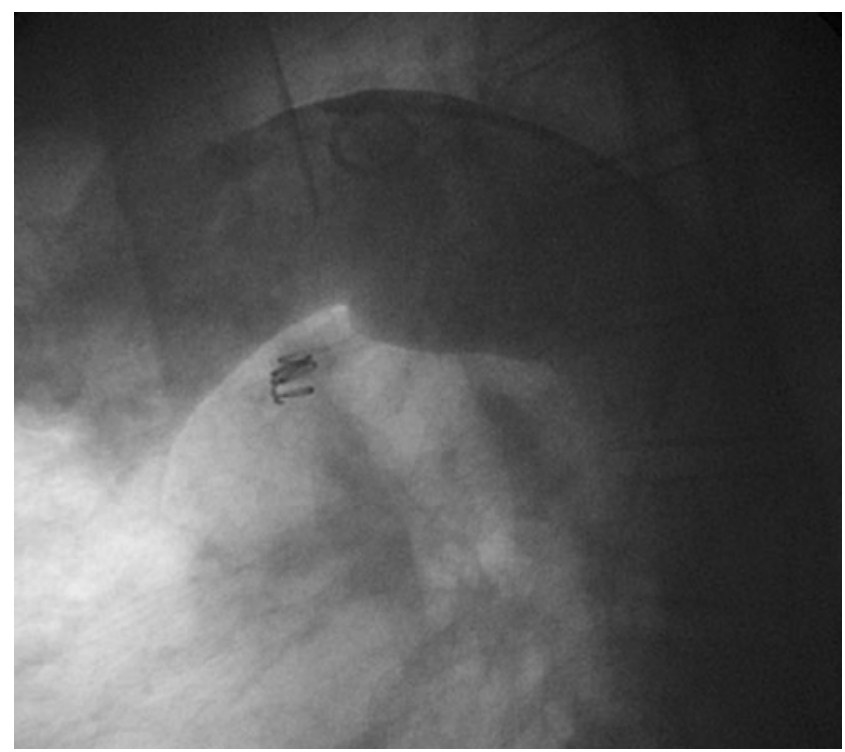

Fig. 4 Angiography of the aortic arch. Aortic arch with proximal end of the PDA containing the occluder coil. The PDA is occluded 


\section{Discussion}

PDA is usually diagnosed shortly after birth, and treated surgically or percutaneously to prevent the development of pulmonary hypertension and heart failure. The two patients described here both reached adulthood with an untreated, significant PDA. The first case represents a more complex form of PDA, associated with additional congenital cardiovascular anomalies and with left ventricular volume overload as one of its main characteristics. In the second patient, pulmonary hypertension was the sole reason for closure of the PDA, and left ventricular dilation had not developed. A difference in shunt size is probably the main reason for this difference. In addition, it may be possible that patient B's severe left ventricular hypertrophy due to hypertension prevented left ventricular dilatation. Closure of a PDA is only feasible when irreversible pulmonary hypertension has not yet developed. In adults, percutaneous closure with a closure device or coil is the preferred treatment [2]. In patient $A$, surgical correction of the PDA was performed because of its large size and concomitant anomalous aortic anatomy. PDA patients who are beyond surgical or percutaneous closure should be treated according to the guidelines for Eisenmenger syndrome. We will report on Eisenmenger syndrome in another issue of this journal.

Open Access This article is distributed under the terms of the Creative Commons Attribution Noncommercial License which permits any noncommercial use, distribution, and reproduction in any medium, provided the original author(s) and source are credited.

\section{References}

1. Schneider DJ, Moore JW. Patent ductus arteriosus. Circulation. 2006; 114:1873-82.

2. Baumgartner H, Bonhoeffer P, De Groot NM, et al. Guidelines for the management of grown-up congenital heart disease (new version 2010): The Task Force on the Management of Grown-up Congenital Heart Disease of the European Society of Cardiology (ESC), endorsed by the Association for European Paediatric Cardiology (AEPC). Eur Heart J. 2010;31:2915-57. 